\title{
Experimental characterization of axillary/underarm interface pressure in swing-through crutch walking
}

\author{
James Borrelli, PhD; ${ }^{*}$ Henry W. Haslach Jr, PhD \\ Department of Mechanical Engineering, University of Maryland-College Park, College Park, MD
}

\begin{abstract}
Supporting weight on the upper support of crutches is not recommended because it can lead to axillary nerve damage. Despite this warning, improper axillary loadings may still occur because of a lack of arm strength or fatigue. It is generally accepted that improper use of conventional axillary crutches contributes to axillary nerve damage, but surprisingly there are no studies characterizing axillary support/underarm configurations. In this study, we compared traditional and horizontal axillary support designs by measuring various biomechanical parameters on the axillary support during a swingthrough gait while supporting weight on the axillary support. Subjects found the axillary support that remains horizontal to be more comfortable than the axillary support of axillary crutches. The higher perceived comfort may be attributed to the lower force and contact area, both average and maximum, developed on the horizontal axillary support and/or shorter excursion of the position of the center of force during a stride. These findings suggest that avoiding all weight bearing on the axillary support may be an overly conservative recommendation for supports that remain horizontal. Individuals with insufficient arm strength may benefit by considering this type of support, but because further study is needed, a physical therapist should be consulted.
\end{abstract}

Key words: ambulation, axillary crutches, axillary force distribution, axillary support, center of force, crutch palsy, crutch walking, Easy Strutter Functional Orthosis System, gait, underarm load.

\section{INTRODUCTION}

Crutches are used to reduce weight bearing on the lower limbs by supporting some weight through the arms in order to compensate for temporary or permanent disabilities [1-2]. Proper crutch use, by supporting loads through the arms, increases the energy expenditure of walking [1,3-7]. The high energy cost may lead some users to support weight through the axillary support, which some evidence suggests is easier, and may also keep others from ambulating upright altogether. Crutch users are advised to support loads on the crutch only through the arms because supporting weight on the axillary support may cause trauma to the axillary nerve or axillary artery injury [8-9]. Located on the top of a conventional axillary crutch is an axillary support that has a pad or cushion covering, whose presence suggests that manufacturers recognize the possibility of improper use and attempt to mitigate discomfort. Even following the prescribed method of crutch use does not preclude possible injury. Prolonged and excessive force on the handgrip may lead to carpal tunnel syndrome [10]. Just as Sala et al. have proven that different handgrip designs can alter

Abbreviations: $\mathrm{ANOVA}=$ analysis of variance, $\mathrm{BW}=$ body weight, $\mathrm{COF}=$ center of force, ESFOS = Easy Strutter Functional Orthosis System.

*Address all correspondence to James Borrelli, PhD; Mechanical Engineering, Baltimore Ave, College Park, MD 20740; 301-405-5338. Email: jrborrelli@gmail.com

http://dx.doi.org/10.1682/JRRD.2012.01.0013 
loading patterns [11], this study investigated the effect of two different axillary supports on user-perceived comfort, total force, contact area, and center of force (COF) excursion.

In this study, subjects supported some of their weight on the axillary support through one stride either on conventional axillary crutches or on the Easy Strutter Functional Orthosis System (ESFOS) (Orthotic Mobility Systems; Kensington, Maryland), which had a support that remained horizontal throughout the stride. Perceived comfort and other biomechanical parameters were measured at the underarm/axillary interface to identify differences. We hypothesized that an axillary support that remains horizontal during a stride would be more comfortable because of the characteristics of the force distribution on the axillary support.

\section{BACKGROUND}

The major design difference between the ESFOS and axillary crutches is the motion of the axillary support. The axillary support of the ESFOS remains horizontal during ambulation (Figure 1). The axillary support of a traditional axillary crutch pivots about the point where it contacts the ground, and consequently, the rigid axillary support rotates with respect to the ground in the sagittal plane.

The axillary supports in each design have two components, a structural support and a cushion. The structural supports of axillary crutches are typically wood or stiff plastic, approximately 6 in. long and 1 in. wide, that deform very little under body weight (BW). The support is covered with a less than 0.5 in. thick rubber cushion. The structural support of the ESFOS is a relatively thin aluminum beam, 0.5 in. wide and 6 in. long and covered with a 1 in. thick polyethylene cushion. The structural support of the ESFOS is pinned at each end, and the support deflects when loaded. The axillary pad of the ESFOS has a larger surface area, $\sim 97 \mathrm{~cm}^{2}$, compared with axillary crutches, $\sim 33 \mathrm{~cm}^{2}$ [12].

Goh et al. investigated underarm loads when improperly using an axillary crutch and found that loads were as high as 34 percent BW [13]. Goh et al. used axial strain gauges mounted on a conventional axillary crutch to estimate the load on the handgrip and axillary support while using a one-leg swing-through gait and improperly supporting weight on the axillary support. Goh et al. point out that proper crutch use requires supporting nearly the entire BW through the arms and that individuals with

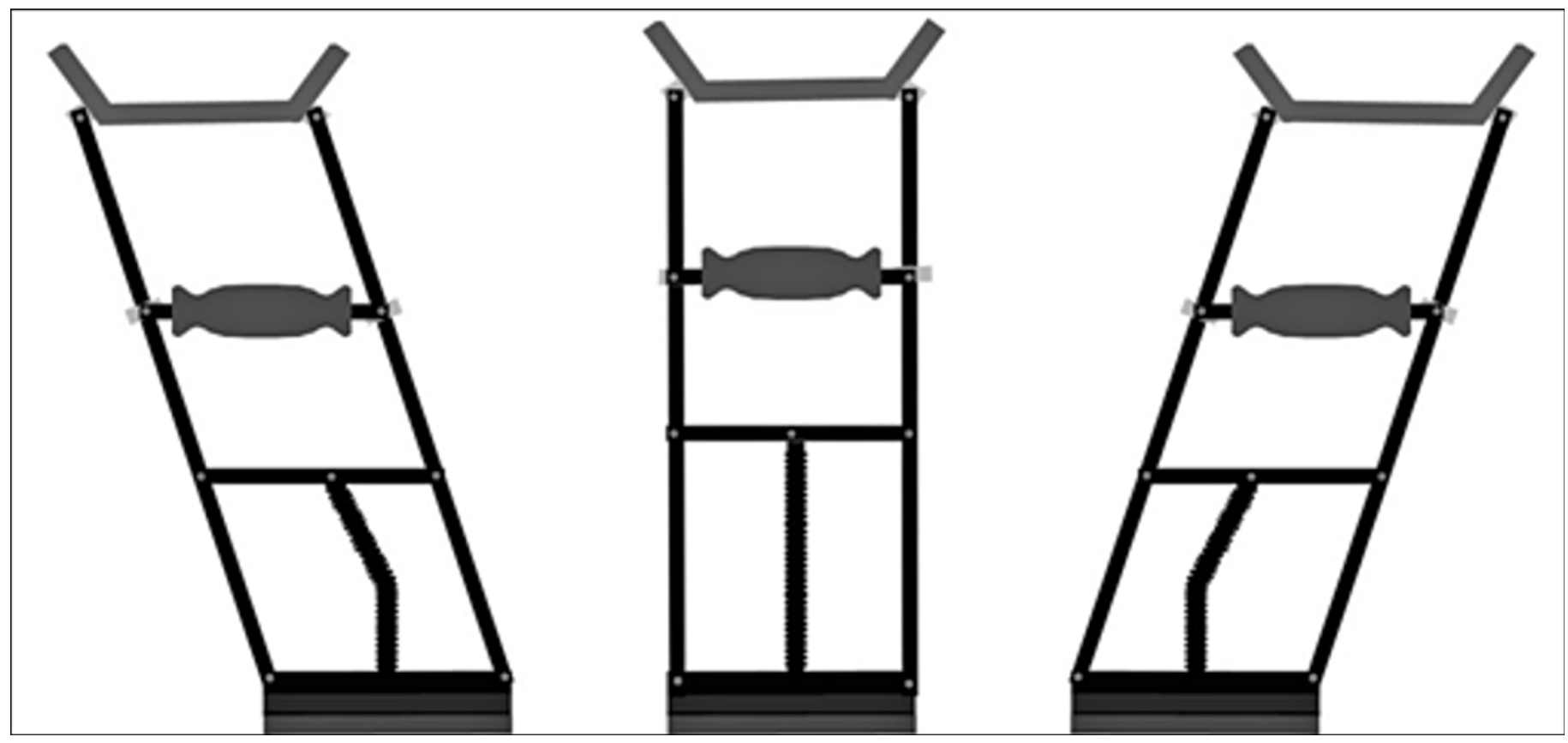

Figure 1.

Easy Strutter Functional Orthosis System assistive device with axillary support that remains level during stride. 
weak upper limbs may resort to supporting weight through the axillary support [13]. Even with the recommended technique, some BW was loaded on the axillary support. Another study by Stallard et al. used a force plate to measure the total load on the crutch (handgrip and possibly axillary support) while using a one-leg swing-through gait [14]. They found loads of 51 percent BW on the lifted leg and 54 percent BW on the landing leg side [14].

The manufacturer of the ESFOS claims that supporting weight on its horizontal axillary support is safe because of the novel design of the ESFOS [15]. Without addressing safety, there is some evidence that supporting weight on the axillary support may be otherwise beneficial. Magee and Kenney measured improved endurance and slightly lower energy expenditure while using the ESFOS compared with Lofstrand crutches [16]. In a case study involving an adolescent with lumbar-level myelodysplasia who required a wheelchair for community ambulation, Magee and Kenney reported a return to upright community ambulation through use of the ESFOS and weight sharing between the handgrip and axillary support [16]. Such a return to upright ambulation may be related to the decrease in energy expenditure through ESFOS use compared with Lofstrand crutches (observed by Nyland et al. [12]). Nyland et al. proved that patients supporting weight on the axillary support of the ESFOS also experienced lowered palmar pressures compared with axillary crutches [12]. Their subjects, who were 1 yr postunilateral knee or hip replacement, used a modified 3point gait and supported the majority of their BW through the underarm while using the ESFOS. The pressure patterns on the handgrips were recorded, but the pressure on the axillary support was not measured.
These studies suggest that supporting some weight on the underarm rather than through the arms makes crutch use seem easier. Magee and Kenney [16] and Nyland et al. [12] did not attempt to measure whether there is some quantifiable difference between the axillary pad of axillary crutches and the ESFOS that allows weight to be supported on the axillary pad of the ESFOS.

\section{METHODS}

\section{Subjects}

Eight nondisabled college-aged students, four men and four women with no history of shoulder trouble (Table 1), served as subjects in this crossover design study.

\section{Data Collection}

Subjects were fit with the ESFOS or axillary crutches with the axillary support $1 \mathrm{in}$. below the underarm and the handgrip adjusted to a height so that the elbow was flexed no more than $30^{\circ}$. Following device fitting, the subjects were given instruction on performing a swingthrough crutch gait. Subjects were allowed to practice until they felt comfortable using the devices. The subjects started from a stopped position and completed one halfgait cycle, the crutch stance phase, before coming to a complete stop. In a swing-through gait, the subject advances both assistive devices to a comfortable distance in front of them and lifts both feet and swings them past the assistive devices, supporting the weight of their body on the axillary support and/or the handgrip. When the

Table 1.

Subject characteristics.

\begin{tabular}{|c|c|c|c|c|c|}
\hline Subject & Sex & $\begin{array}{l}\text { Age } \\
\text { (yr) }\end{array}$ & $\begin{array}{c}\text { Height } \\
\text { (in.) }\end{array}$ & $\begin{array}{c}\text { Weight } \\
\text { (lb) }\end{array}$ & Handedness \\
\hline 2 & M & 22 & 71.25 & 161 & $\mathrm{R}$ \\
\hline 4 & M & 21 & 67.25 & 188 & $\mathrm{R}$ \\
\hline 5 & $\mathrm{~F}$ & 22 & 64.75 & 132 & $\mathrm{R}$ \\
\hline 8 & $\mathrm{~F}$ & 22 & 67.50 & 202 & $\mathrm{R}$ \\
\hline Mean \pm SD & - & $22.8 \pm 1.3$ & $67.50 \pm 3.01$ & $163.38 \pm 30.26$ & - \\
\hline
\end{tabular}

$\mathrm{F}=$ female, $\mathrm{M}=$ male, $\mathrm{R}$ = right, $\mathrm{SD}$ = standard deviation. 
subject's feet come in contact with the ground, the subject comes to a complete stop and ceases weight bearing on the axillary support and/or the handgrip.

Each subject performed three strides using axillary crutches with hand support, axillary crutches without hand support, the ESFOS with hand support, and the ESFOS without hand support. The order in which the subjects used each device and gait (with or without hand support) was chosen at random. The subjects, who had never used crutches previously, were advised for the trials with hand support to support a comfortable weight on the axillary support of either the ESFOS or axillary crutches while simultaneously supporting weight on the handgrip. The trials involving weight support through the axillary support without hand support are intended to identify biomechanical parameters that may not be present when hand support is used. For example, users may have a natural tendency to support more weight on the handgrip of the one device over the other, which would develop less load on one of the axillary supports.

Forces on each axillary support were measured using a thin compliant sensor (F-Scan sensor \#9811, Tekscan; Boston, Massachusetts). The F-Scan sensor system uses a grid of resistive ink sandwiched between two sheets of Mylar. The sensor has 16 rows and 6 columns, forming 96 individual force sensors with a density of 4 sensors per square inch. The F-Scan sensor measures forces that are normal to the surface of the polyester sheet. The data are output through the accompanying software, which also calculates the location of the COF, total force, and contact area.

Sensors were affixed to the axillary pads of an axillary crutch (Figure 2(a)) and the ESFOS (Figure 2(b)) using double-sided tape. Prior to every test, the sensors were calibrated using a force platform. The subjects were instructed to load the force plate to $35 \mathrm{lb}$ by applying force only to the axillary pad while avoiding squeezing the sides of the pad. Upon completion of the tests, subjects were asked to rate the comfort and stability of axillary crutches and the ESFOS on a scale from 1 to 10, with 10 representing the most comfortable and the most stable during the stride. Each participant rated, in particular, the comfort level of the axillary pad of the ESFOS or axillary crutches during a stride while supporting weight on the axillary support and also rated the stability of the overall device during a stride.

\section{Statistical Analysis}

A series of repeated measures analysis of variance (ANOVA) tests was used to determine statistically significant differences between devices for the parameters, maximum force, average force, maximum contact area, average contact area, and COF location during various points in the step. Subjects with incomplete data sets were removed from the cohort. A probability level of 0.05 with Bonferroni corrections for multiple comparisons $(0.05 / 30=$ $0.00167)$ was used to indicate statistical significance. The Bonferroni correction is used to reduce the likelihood of

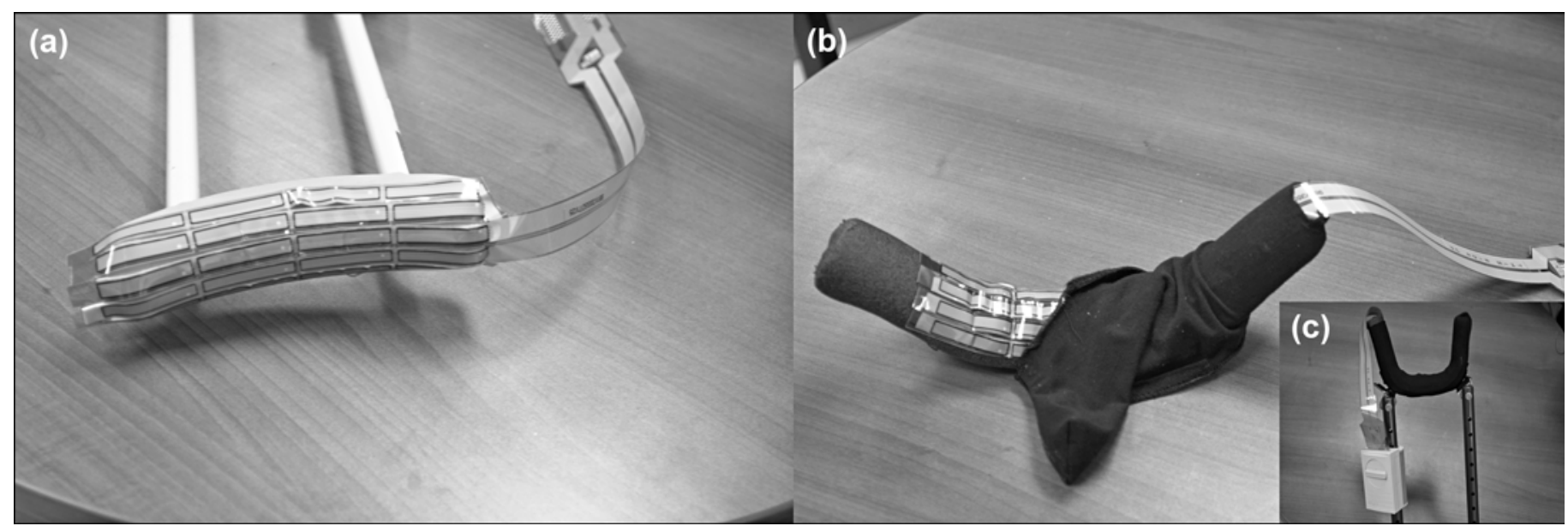

Figure 2.

(a) Axillary crutch pad with F-Scan pressure sensor taped to cushion. (b) Easy Strutter Functional Orthosis System (ESFOS) axillary support cushion with F-Scan pressure sensor attached. (c) ESFOS with F-Scan pressure sensor mounted. 
incorrectly rejecting the null hypothesis, that there is no difference between the sample means. The four treatments, axillary crutches with hand support, axillary crutches without hand support, ESFOS with hand support, and ESFOS without hand support, were tested for statistically significant differences. If a statistically significant difference was identified, axillary crutches and ESFOS with hands and axillary crutches and ESFOS without hands were tested for statistically significant differences.

\section{RESULTS}

\section{Perceived Comfort Index}

This study measures user-perceived comfort of two assistive devices, one with a conventional axillary support and one that remains horizontal during a stride. The subjects in this study perceived the ESFOS, the device with the axillary support that remains horizontal, as more comfortable (Table 2). The biomechanical parameters that appear to influence user-perceived comfort are the total force on the axillary support, the number of maximum contained in the time history of the total force, and the position of the COF during a stride while using crutches and the ESFOS.

\section{Total Force on Support}

Since the time histories of the two devices fall into one of two categories, it is helpful to report the qualitative features. The two most commonly observed time histories of the equivalent total force exerted on the axillary support had either a single or double peak (Figure 3). More than 95 percent of the time histories measured at the axillary pad could be qualitatively described by one of the two curves shown in Figure 3.

Table 3 summarizes the number of local maxima and which local maximum was largest. When using axillary

Table 2.

Average comfort and stability score of axillary crutches and Easy Strutter Functional Orthosis System (ESFOS) as perceived by study participants.

\begin{tabular}{lc}
\hline \multicolumn{1}{c}{ Device } & Average Score \\
\hline ESFOS Comfort & 5.9 \\
Axillary Crutch Comfort & 4.7 \\
ESFOS Stability & 6.3 \\
Axillary Crutch Stability & 6.0 \\
\hline \hline
\end{tabular}

crutches, there was a nearly 50-50 split between one and two local maxima, whereas the ESFOS had a 70-30 split. Less than 5 percent of the time histories had three or more local maxima. The first maximum was most frequently measured to be the largest local maximum during a stride whether the ESFOS or axillary crutches was used with or without hand support.

To further characterize the behavior of the total force, Table 4 lists the average duration of the force on the axillary support and point in the gait cycle when the maximum force occurs. The position 50 percent corresponds to completion of half of the stride. This may or may not correspond to when the assistive device is in the vertical orientation. The maximum force occurs later in the stride when using the ESFOS.

The four treatments, axillary crutches with and without hand support and ESFOS with and without hand support, were tested for statistically significant differences. The calculated $p$-value was below the critical $p$-value with Bonferroni correction for multiple comparisons (0.05/30), indicating statistically significant differences between the maximum total force $(<0.001)$, average total force $(<0.001)$, maximum contact area $(<0.001)$ and average contact area $(<0.001)$ for the four treatments. The test does not tell us which of the values is different from the rest, so we further compared the ESFOS and axillary crutch with hand support and then the ESFOS and axillary crutches without hand support. The interaction effects and within-subjects differences were not statistically significant for any of the tests in Table 5 or $\mathbf{6}$.

Table 5 compares the average values and standard deviations for the maximum total force, average total force, maximum contact area, and average contact area with hand support. The given $p$-values are for a pairwise ANOVA. Lower maximum total force and maximum contact area were measured when subjects used the ESFOS compared with axillary crutches. The difference in average force and also average contact area were smaller for the ESFOS but not statistically significant at this alpha level.

Table 6 compares the same values as Table 5 when subjects used a swing-through gait without hand support. Lower maximum total force and average total force were measured when subjects used the ESFOS compared with axillary crutch without hand support. The average contact area of the ESFOS is lower than axillary crutches, again, but is not statistically significant at this level. 


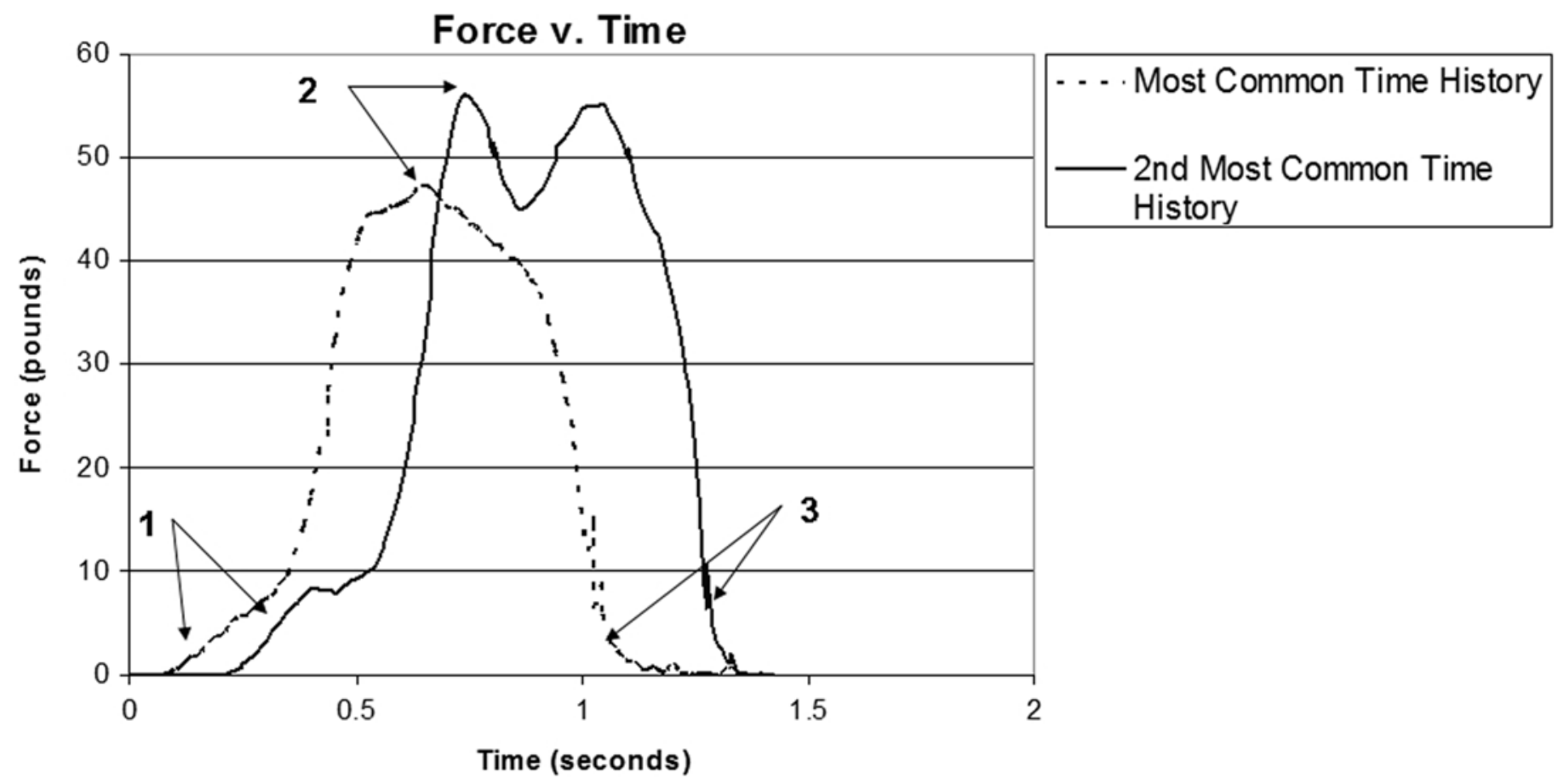

Figure 3.

Two most common time histories of total force on axillary support. Majority of time histories could be described qualitatively by two curves shown, either having one maxima or two. Numbers 1, 2, 3 represent three discreet points in stance phase, point 2 is time when maximum force is recorded. Points 1 and 3 are times when $10 \%$ of maximum force is measured.

Table 3.

Percentage of trials in which time varying force had 1, 2, or more peaks and also particular peak at which absolute maximum force occurs. Values are rounded to nearest integer percent.

\begin{tabular}{|c|c|c|c|c|}
\hline Measure & $\begin{array}{l}\text { Axillary Crutches } \\
\text { with Hands }\end{array}$ & $\begin{array}{l}\text { Axillary Crutches } \\
\text { without Hands }\end{array}$ & $\begin{array}{c}\text { ESFOS } \\
\text { with Hands }\end{array}$ & $\begin{array}{c}\text { ESFOS } \\
\text { without Hands }\end{array}$ \\
\hline $\begin{array}{l}\text { Number of Peaks (\% with } 1 \text { peak/\% with } \\
2 \text { peaks/\% with } 3 \text { or more peaks) }\end{array}$ & $46 / 48 / 6$ & $60 / 33 / 7$ & $73 / 22 / 4$ & $55 / 43 / 2$ \\
\hline
\end{tabular}

Table 4.

Mean values and standard deviation for duration of load and point in gait cycle when maximum force was measured given as percent of stride completed.

\begin{tabular}{lcccc}
\hline \multicolumn{1}{c}{ Measure } & $\begin{array}{c}\text { Axillary Crutches } \\
\text { with Hands }\end{array}$ & $\begin{array}{c}\text { ESFOS } \\
\text { with Hands }\end{array}$ & $\begin{array}{c}\text { Axillary Crutches } \\
\text { Without Hands }\end{array}$ & $\begin{array}{c}\text { ESFOS } \\
\text { Without Hands }\end{array}$ \\
\hline Duration of Load (s) & $0.99 \pm 0.38$ & $0.97 \pm 0.30$ & $1.05 \pm 0.26$ & $0.78 \pm 0.20$ \\
Position of Stride at Maximum Force (\%) & $44.44 \pm 16.53$ & $51.50 \pm 18.64$ & $54.97 \pm 18.64$ & $51.97 \pm 13.67$ \\
\hline ESFOS = Easy Strutter Functional Orthosis System. & & & & \\
\hline \hline
\end{tabular}


Table 5.

Average values of maximum total force, average total force, maximum contact area, and average contact area with hand support.

\begin{tabular}{|c|c|c|c|}
\hline Measure & $\begin{array}{l}\text { Axillary Crutches with Hands } \\
\text { (mean } \pm \text { SD) }\end{array}$ & $\begin{array}{l}\text { ESFOS with Hands } \\
\quad(\text { mean } \pm \text { SD) }\end{array}$ & $p$-Value \\
\hline$\overline{\text { Maximum Force (lb) }}$ & $30.23 \pm 14.72$ & $18.21 \pm 12.65$ & $<0.001^{*}$ \\
\hline Average Force (lb) & $14.99 \pm 10.54$ & $9.00 \pm 8.41$ & 0.01 \\
\hline Average Contact Area (in. ${ }^{2}$ ) & $6.77 \pm 3.26$ & $4.61 \pm 3.78$ & 0.01 \\
\hline
\end{tabular}

${ }^{*}$ Below critical $p$-value with Bonferroni correction, 0.05/30.

ESFOS = Easy Strutter Functional Orthosis System, SD = standard deviation.

Table 6.

Average values of maximum total force, average total force, maximum contact area, and average contact area without hand support.

\begin{tabular}{|c|c|c|c|}
\hline Measure & $\begin{array}{l}\text { Axillary Crutches without Hands } \\
\text { (mean } \pm \text { SD) }\end{array}$ & $\begin{array}{l}\text { ESFOS without Hands } \\
\quad(\text { mean } \pm \text { SD) }\end{array}$ & $p$-Value \\
\hline Average Force (lb) & $22.45 \pm 7.08$ & $16.00 \pm 5.16$ & $<0.001^{*}$ \\
\hline Average Contact Area (in. ${ }^{2}$ ) & $9.50 \pm 2.52$ & $8.14 \pm 2.76$ & 0.04 \\
\hline
\end{tabular}

*Below critical $p$-value with Bonferroni correction, 0.05/30.

ESFOS = Easy Strutter Functional Orthosis System, SD = standard deviation.

The total force described in the previous section is distributed over the axillary support. Three areas of higher relative force were consistently observed on the support (Figure 4) whether the ESFOS or axillary crutches were used with or without hand support. The areas of high force were typically where the front of the rib cage, back of the rib cage, and the upper arm contact the top and sides of the axillary support; these areas are circled in Figure 4. Some load is on the top of the support and some is on the sides. The inner box represents the area that is parallel to the ground when axillary crutches are held vertical, and at all times during a stride with the ESFOS. The outer box is where the cushion and sensor slope downward.

The position of the COF on the axillary supports was recorded at three times during each stride. Figure 5 plots the position of the COF at times 1, 2, and 3 (from Figure 3) when using the ESFOS or axillary crutches with or without hand support. The numbers 1, 2, and 3 denote times during the stride when 10 percent of the maximum force was recorded, when the maximum force was recorded, and the second time when 10 percent of the maximum force was recorded just before the end of the stride. The location of the COF at the three points in time is shown in Figure 5.

The positive $y$-axis is the direction of travel and the positive $x$-axis is toward the body while the negative $x$ -

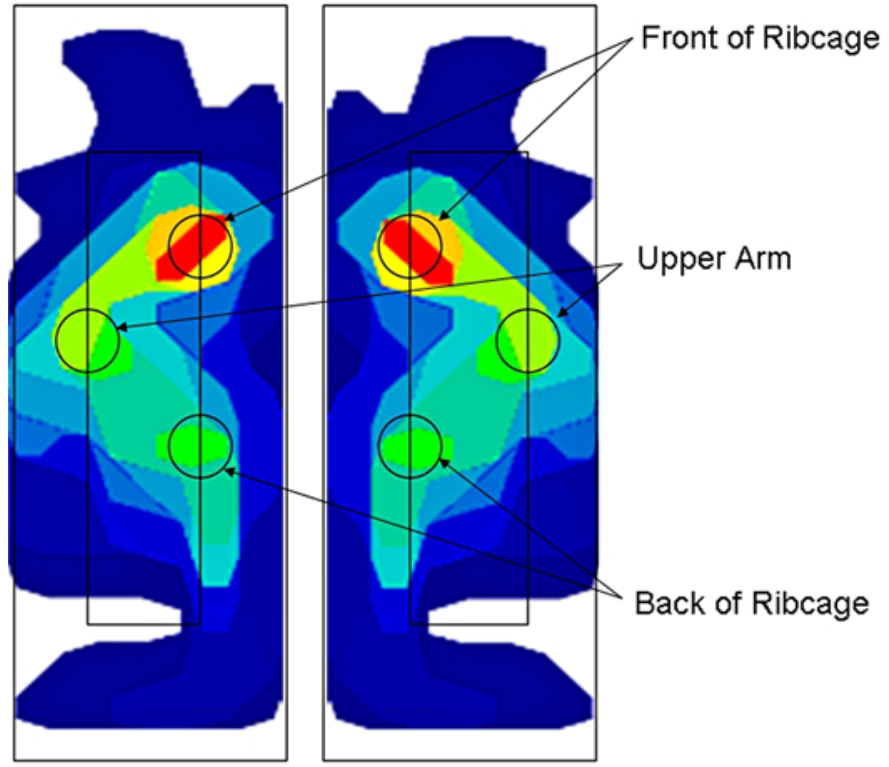

Figure 4.

Typical force distribution on left and right axillary support. Areas of high force are in red and low force in blue. Inner box represents hard support under cushion of each axillary support and portion in outer box is where cushion curves downward.

axis is away from the body, toward the arm. This choice of axes assumes that the right and left sides are symmetrical. Three points are not enough to definitively say where the 


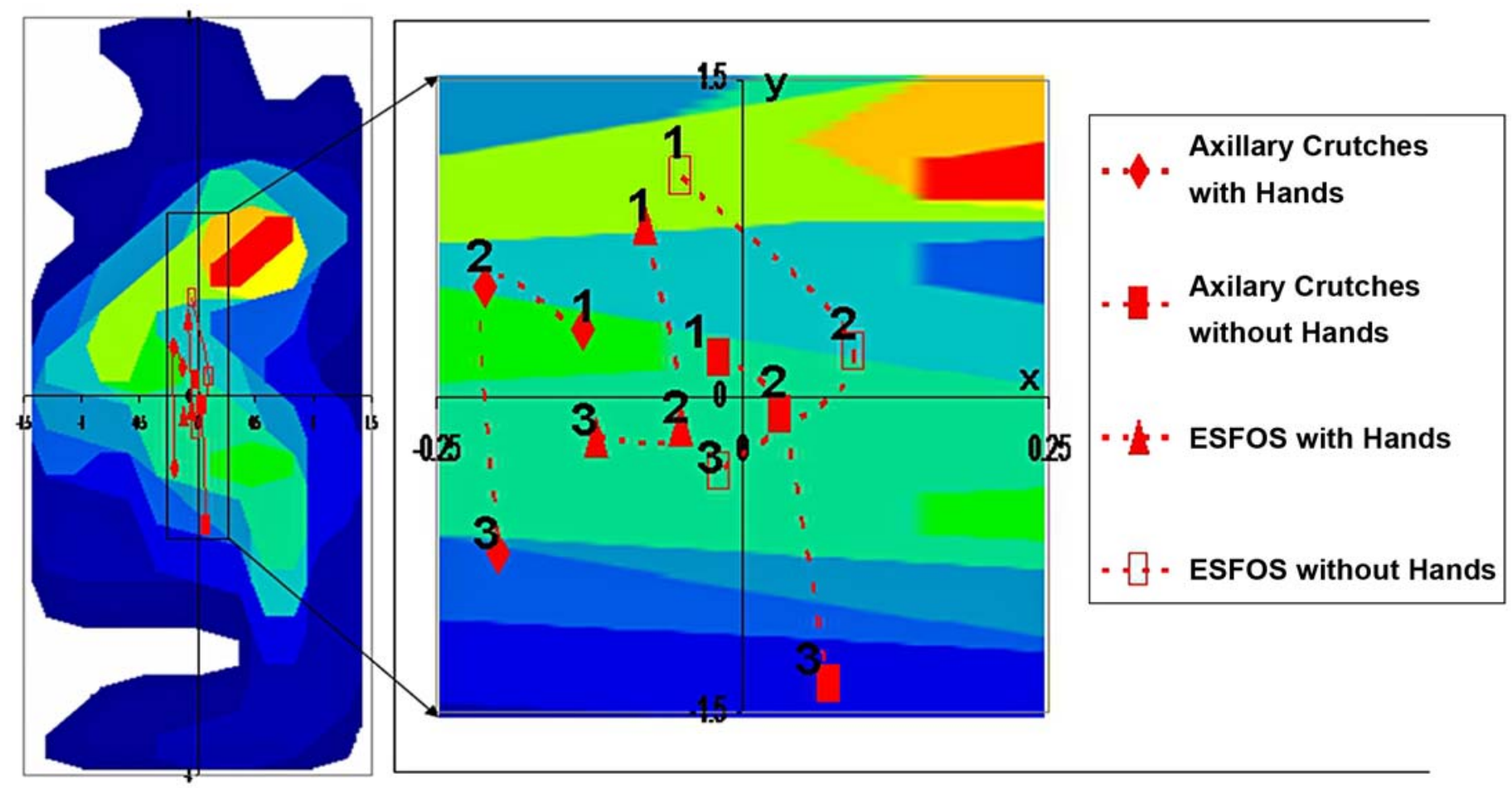

Figure 5.

Trajectory of center of force on left axillary pad while using Easy Strutter Functional Orthosis System (ESFOS) or axillary crutches with or without hand support. Diamond, solid square, triangle, and square outlines are axillary crutches with hand support, axillary crutches without hand support, ESFOS with hand support, and ESFOS without hand support, respectively. Graph on right is close up of boxed area in figure on left. Points 1, 2, and 3 are times when maximum force occurs (2) and when 10\% of maximum occurs (1 and 3).

COF is at each point of the entire stride; however, some general trends were observed. The excursion of the COF is from front to back. Removing hand support increases the $y$-coordinate range (1.27 to 1.55 in. for axillary crutches and 1.02 to $1.40 \mathrm{in}$. for the ESFOS) of the COF and the $x$-coordinate domain (from 0.07 to $0.09 \mathrm{in}$. for axillary crutches and from 0.07 to 0.14 in. for the ESFOS). The use of hands produces a shorter excursion on both crutch types. The distance the COF travels through, assuming a linear trajectory on the axillary pad/ underarm manifold, is 1.49 in., 1.49 in., 1.05 in., and 1.42 in. for axillary crutches with hand support, axillary crutches without hand support, ESFOS with hand support, and ESFOS without hand support, respectively.

The $x$ - and $y$-coordinates of the COF were compared at three different times (1, 2, and 3 from Figure 3) for the four treatments. The ANOVA test indicates no statistically significant difference between the $x$-position of the COF at times 1 , 2, or 3 for the four treatments. The $p$-values were not below the critical value between the $x$-coordinates at positions 1 (0.31), $2(0.11)$, or 3 (0.019). There was a statistically significant difference between the $y$-position of the COF for the four treatments. The $p$-values were below the critical value with Bonferroni correction (0.05/30) at positions $1(<0.001), 2(<0.001)$, and $3(<0.001)$. Since the test does not indicate which treatment is different from the rest, we further compared the $y$-position of the COF of the ESFOS and axillary crutches with hand support (Table 7) and then the ESFOS and axillary crutches without hand support (Table 8). The interaction effects and within subjects differences were not statistically significant for any of the comparisons.

The difference in $y$-position at the three points for axillary crutches and ESFOS with hand support is statistically significant at the end of the stride, point 3 (Table 7). But for axillary crutches and the ESFOS without hand 
Table 7.

Position of center of force during swing-through gait with hand support.

\begin{tabular}{|c|c|c|c|c|}
\hline Time Point & Coordinate & $\begin{array}{l}\text { Axillary Crutches with Hands } \\
\text { (in., mean } \pm \text { SD) }\end{array}$ & $\begin{array}{l}\text { ESFOS with Hands } \\
\text { (in., mean } \pm \text { SD) }\end{array}$ & $p$-Value \\
\hline \multirow[t]{2}{*}{1} & $x$ & $-0.11 \pm 0.45$ & $-0.03 \pm 0.56$ & Not Compared \\
\hline & $y$ & $0.13 \pm 0.94$ & $0.86 \pm 1.31$ & 0.004 \\
\hline & $y$ & $0.52 \pm 0.87$ & $-0.04 \pm 1.06$ & 0.01 \\
\hline \multirow[t]{2}{*}{3} & $x$ & $-0.16 \pm 0.47$ & $-0.16 \pm 0.58$ & Not Compared \\
\hline & $y$ & $-0.92 \pm 0.72$ & $-0.24 \pm 0.97$ & $<0.001^{*}$ \\
\hline
\end{tabular}

Table 8.

Position of center of force during swing-through gait without hand support.

\begin{tabular}{|c|c|c|c|c|}
\hline Time Point & Coordinate & $\begin{array}{l}\text { Axillary Crutches without Hands } \\
\text { (in., mean } \pm \text { SD) }\end{array}$ & $\begin{array}{l}\text { ESFOS without Hands } \\
\quad \text { (in., mean } \pm \text { SD) }\end{array}$ & $p$-Value \\
\hline \multirow[t]{2}{*}{1} & $x$ & $0.07 \pm 0.27$ & $-0.01 \pm 0.32$ & Not Compared \\
\hline & $y$ & $0.06 \pm 0.64$ & $1.08 \pm 0.87$ & $<0.001^{*}$ \\
\hline 2 & $y$ & $-0.17 \pm 0.51$ & $-0.12 \pm 1.09$ & 0.86 \\
\hline 3 & $x$ & $0.13 \pm 0.29$ & $0.01 \pm 0.53$ & Not Compared \\
\hline
\end{tabular}

*Below critical $p$-value with Bonferroni correction, 0.05/30.

ESFOS = Easy Strutter Functional Orthosis System, SD = standard deviation.

support, the difference between the $y$-position of the COF at both the beginning and end of the stride, points 1 and 3 (Table 8), is statistically significant.

The COF generally moves from the anterior to the posterior end of the axillary support in the opposite direction of ambulation. In Figure 5, the trajectory for the trial without hands is moved closer to the trunk than the trajectory for the trial with hands for both types of crutch. The trajectories for the conventional crutch are both closer to the upper arm than the respective trajectories for the ESFOS. The hands-free trajectories for both devices are monotonic, while in the trials with hands the trajectory for the conventional crutch changes direction from the first portion of the stride to the last portion. The trajectory for the ESFOS with hands moves slightly closer to the arm at the end of the stride.

\section{DISCUSSION}

We hypothesized that the characteristics of the force distribution of the ESFOS make it more comfortable. The factors that appear to be determinants of user comfort are total force, number of maximum, pressure, and/or COF excursion. Other studies show decreases in energy expenditure and palmar pressure and a return to community ambulation through axillary weight bearing on the ESFOS as well as a preference for the ESFOS [12,16]. The preference of the subjects of this study for the ESFOS compared with axillary crutches while supporting some weight on the axillary support likely has a physical basis in the force behavior on the support. The lower total maximum and average force on the ESFOS and shorter excursion of the COF may explain how the horizontal support improves user perceived comfort.

Magee and Kenney [16] and Nyland et al. [12] argue that the oversized cushion area on the ESFOS compared with axillary crutches allows underarm pressure to be distributed over a larger area. Surprisingly, despite having an available surface area larger than axillary crutches, users of the ESFOS utilized both a smaller average and maximum contact area than with axillary crutches in this study. However, lower average force and average contact area were developed, although this was not statistically significant. Despite this fact, the resulting average pressure, calculated by dividing the average force and contact area, for the ESFOS with hand support was 1.95 psi and 2.21 psi for axillary crutches with hand support. 
The number of peak forces achieved and their magnitude may also be related to perceived comfort. When using the ESFOS, a single peak, like in Figure 3, was more frequently exhibited, whereas when using axillary crutches, a double peak was more frequently exhibited. It seems it is not uncommon for both single and double peaks to occur with crutch use because both types of curves have been reported previously when using a swing-through gait [13-14]. The initial maximum force on the rotating support of the conventional crutch, when hands are used, occurs prior to midstride, and the second maximum occurs just afterward. Compared with a single maximum, a double maximum may cause the crutch to slow down and then speed up, which would result in larger forces on the axillary support to stabilize the device, which may be indicated by the slightly greater perceived stability of the ESFOS.

Despite the limitations of this study, we believe that the horizontal axillary support of the ESFOS leads to less shifting of the underarm. Furthermore, supporting some weight on the axillary support reduces the need to support weight on the handgrip. This type of weight sharing may decrease the risk of carpal tunnel syndrome by lowering planar pressure, which reduces carpal tunnel pressure [10]. The motion of the COF when using axillary crutches and the ESFOS moves from the trunk toward the upper arm from points 1 to 2 and 2 to 3 (Figure 5). We cannot say for sure, but this may be when the maximum force occurs, which is slightly before midstride and slightly afterward for axillary crutches and the ESFOS with hand support, respectively. The side-to-side motion may be exerting a shear force on the axillary nerve or other structures in the underarm region. The ESFOS should be considered if an individual is too weak for conventional crutches, is developing carpal tunnel syndrome, or needs more stability.

The maximum force on the axillary crutches was higher than the ESFOS both with and without hand support. When the subjects performed a stride without hand support, the smallest maximum force that could have possibly been developed on the axillary support would be the sum of BW and inertia. However, the fact that the maximum force was higher on axillary crutches than the ESFOS indicates that axillary crutches require additional forces to stabilize the crutch or body, which again may be indicated by the slightly greater perceived stability. The larger excursion of the COF may partially explain the need for extra force on the axillary support of axillary crutches. The shorter COF excursion experienced by ESFOS users suggests that the horizontal support may reduce shear forces by keeping the underarm from sliding on the axillary support. Loads on the support may generate a friction force, or more generally a shear force, if the underarm shifts on the axillary pad. This possibility is verified by performing a force balance, assuming impending slip (Figure 6):

$$
\begin{aligned}
& \sum F_{x}=0: G R F_{x}-2 F_{z x}=0, \\
& \sum F_{y}=0: G R F_{y}-F_{z y}-2 F_{z x}=0, \\
& \sum F_{z}=0: G R F_{y}-F_{z}-2 F_{x z}=0 .
\end{aligned}
$$

Resting on the axillary support develops a vertical force, $F_{Z}$, while squeezing applies a normal force, $F_{X}$, to the sides of the axillary pad. The ground reaction force is given as $G R F_{z}$. If the underarm shifts forward or backward, up or down on the support, shear forces may be developed, $F_{Z X}$, $F_{z y} F_{x y}$ or $F_{x z}$. Shear forces were not measured in this

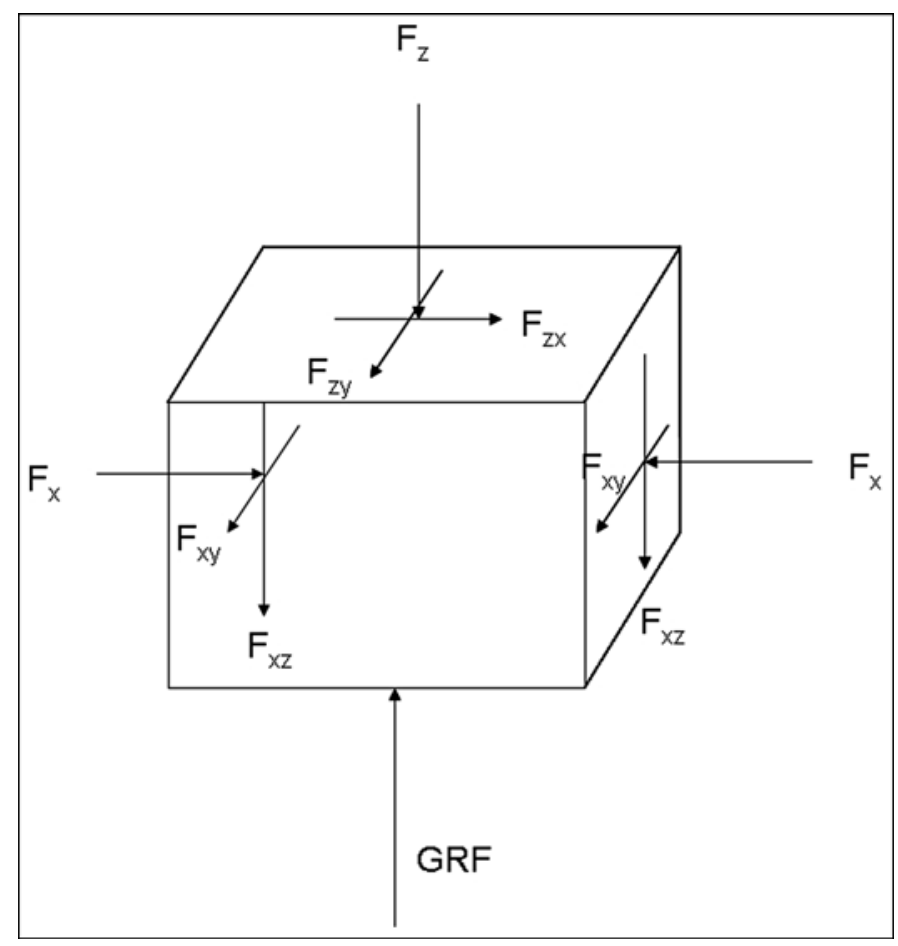

Figure 6.

Diagram idealizes loading on axillary support viewed from front. $F=$ force, $G R F=$ ground reaction force. 
experiment and likely have an effect on the perceived comfort and stability of the axillary supports.

The exact location of the maximum force on the underarm that creates the most risk of injury to the axillary nerve or artery is not known. Deciding whether or not the horizontal support of the ESFOS reduces axillary nerve or artery damage must await further studies on how the forces from an axillary support actually cause injury. The fact that the trajectories for the COF on the conventional crutch are closer to the upper arm indicates that part of the increased discomfort may result from the edge of the conventional support digging into the arm, which would explain numbness and tingling in the arm after supporting weight on the axillary support of axillary crutches, a symptom of axillary nerve damage.

\section{LIMITATIONS}

This experiment provides evidence that there is a difference between the axillary support of axillary crutches and the horizontal support of the ESFOS, which may allow more weight support than axillary crutches. However, experimental error and low statistical power limit the application of axillary weight sharing with a horizontal axillary support. The cohort and experimental motions represent a rather small portion of the population and range of motions used in daily life. The cohort was rather homogeneous, 21 to $25 \mathrm{yr}$ old nondisabled individuals in good physical shape, and the crutch stance phase is only one specific portion of the gait. Furthermore, all of the subjects in the trial were unfamiliar with crutch walking, and some variation in outcome measures may have been a result of inexperience.

One pair of axillary crutches and one pair of ESFOS were used in the experiment. The sensors used on each assistive device were not replaced during the course of testing. The sensors were cut into parallel columns and secured to the axillary supports with double-sided tape. In some trials, the double-sided tape began to break free toward the extreme front and back of the axillary pad of both devices. This allowed the F-Scan sensor to fold on itself. The worst case observed had three sensors stacked on top of one another in a corner of the ESFOS's axillary pad. Each sensor has an area of $0.25 \mathrm{in}^{2}$, so three sensors overlapping each other results in an error of $0.5 \mathrm{in} .^{2}$. The ESFOS sensor was consistently in worse shape than those on the axillary crutches and likely resulted in a larger than actual contact area being measured. If this were true for all trials, the average pressure on the ESFOS would be approximately equal to axillary crutches. Another limitation of the F-Scan system is the rise time when subjected to a step load, which is made worse when covered with felt [17]; for this reason, more emphasis should be placed on relative differences rather than quantitative values [18]. However, the axillary loading duration was at least twice the dynamic response time reported by Sumiya et al. [17] for both assistive devices.

Another limitation is the large number of multiple comparisons in this study, which reduces the power of the statistics. Performing an additional experiment with fewer multiple comparisons may result in statistically significant differences. For example, the average force was not statistically significant between the ESFOS and axillary crutches while the handgrip was used. The $p$ value was small but not below the threshold defined in this study because of the multiple comparisons. If another experiment were performed with a new cohort only comparing the average force of the two axillary supports while using the handgrip, it is possible that the difference would be statistically significant. Further testing with fewer outcome measures and/or fewer multiple comparisons and a more diverse cohort is indicated.

The samples were tested for normality, and not all samples were normal according to the Shapiro-Wilk test. Only the ESFOS with hands and without hands samples for maximum force samples and axillary crutches with hand for maximum contact area samples were normally distributed. A consequence of using a non-normally distributed sample with the repeated measures ANOVA is that the likelihood of a false positive increases. The locations of the COF samples were normally distributed except for the $y$-position of the COF at time 3 for the ESFOS. Ultimately, this means that the statistically significant difference between the $y$-position of the COF of the ESFOS and axillary crutches without hand use at time 3 may be suspect.

\section{CONCLUSIONS}

Best practice recommends against supporting weight on the axillary support of crutches. Despite this fact, the majority of axillary crutches have a pad or cushion covering the axillary support. This suggests that manufacturers recognize users may still support weight on the axillary 
support. It has been suggested that improper usage is due to fatigue or because it is easier; this type of crutch use may increase user endurance. More comfortable axillary support designs may allow for weight sharing between the underarm and handgrip. Weight sharing may reduce the likelihood of cessation of crutch walking, an important consideration since crutch walking rather than wheelchair use positively affects the health of individuals who rely on assistive devices.

In this study, users of the ESFOS perceived an increase in comfort, which may be attributed to lower axillary force, lower pressure, fewer total force maxima, and/or a shorter COF trajectory, which may reduce shifting on the axillary support. Although not all of the differences were statistically significant, a difference was perceived by the user and further study is indicated. Furthermore, physical therapists should consider a weightsharing technique between the axillary support and handgrip for use with the ESFOS on a case by case basis while constantly being aware of the early warning signs of axillary nerve damage onset.

\section{ACKNOWLEDGMENTS}

\section{Author Contributions:}

Study concept and design: J. Borrelli, H. W. Haslach.

Acquisition of data: J. Borrelli.

Analysis and interpretation of data: J. Borrelli, H. W. Haslach.

Drafting of manuscript: J. Borrelli.

Critical revision of manuscript for important intellectual content:

H. W. Haslach.

Statistical analysis: J. Borrelli.

Obtained funding: H. W. Haslach.

Administrative, technical, or material support: J. Borrelli,

H. W. Haslach.

Study supervision: H. W. Haslach.

Financial Disclosures: The authors have declared that no competing interests exist.

Funding/Support: This material was based on work supported by an award from Maryland Industrial Partnerships (MIPS Orthotic Mobility \#2804). The experiment was performed in the University of Maryland Smart Materials and Structures Research Center laboratory, Prof. Amr Baz, Director.

Institutional Review: This study was approved by the University of Maryland-College Park Institutional Review Board (IRB Protocol Number: 05-0596, which includes the informed consent form).

Participant Follow-Up: The authors have no plans to notify the study subjects of the publication of this article because of a lack of contact information.

\section{REFERENCES}

1. Bhambani Y, Clarkson H. Acute physiologic and perceptual responses during three modes of ambulation: walking, axillary crutch walking, and running. Arch Phys Med Rehabil. 1989;70(6):445-50. [PMID:2730307] http://dx.doi.org/10.1016/0003-9993(89)90004-X

2. Gray FB, Gray C, McClanahan JW. Assessing the accuracy of partial weight-bearing instruction. Am J Orthop. 1998; 27(8):558-60. [PMID:9732078]

3. Dounis E, Rose GK, Wilson RS, Steventon RD. A comparison of efficiency of three types of crutches using oxygen consumption. Rheumatol Rehabil. 1980;19(4):252-55.

[PMID:7209290]

http://dx.doi.org/10.1093/rheumatology/19.4.252

4. LeBlanc MA, Carlson LE, Nauenberg T. A quantitative comparison of four experimental axillary crutches. J Prosthet Orthot. 1993;5(1):20-28. http://dx.doi.org/10.1097/00008526-199301000-00007

5. Sankarankutty M, Stallard J, Rose GK. The relative efficiency of "swing through" gait on axillary, elbow and Canadian crutches compared to normal walking. J Biomed Eng. 1979;1(1):55-57. [PMID:537334] http://dx.doi.org/10.1016/0141-5425(79)90011-6

6. Thys H, Willems PA, Saels P. Energy cost, mechanical work and muscular efficiency in swing-through gait with elbow crutches. J Biomech. 1996;29(11):1473-82.

[PMID:8894928]

http://dx.doi.org/10.1016/0021-9290(96)84543-X

7. Wilson JF, Gilbert JA. Dynamic body forces on axillary crutch walkers during swing-through gait. Am J Phys Med. 1982;61(2):85-92. [PMID:7072838] http://dx.doi.org/10.1097/00002060-198204000-00003

8. Feldman DR, Vujic I, McKay D, Callcott F, Uflacker R. Crutch-induced axillary artery injury. Cardiovasc Intervent Radiol. 1995;18(5):296-99. [PMID:8846468] http://dx.doi.org/10.1007/BF00203679

9. McFall B, Arya N, Soong C, Lee B, Hannon R. Crutch induced axillary artery injury. Ulster Med J. 2004;73(1): 50-52. [PMID:15244127]

10. Cobb TK, An KN, Cooney WP. Externally applied forces to the palm increase carpal tunnel pressure. J Hand Surg Am. 1995;20(2):181-85. [PMID:7775748] http://dx.doi.org/10.1016/S0363-5023(05)80004-8

11. Sala DA, Leva LM, Kummer FJ, Grant AD. Crutch handle design: effect on palmar loads during ambulation. Arch Phys Med Rehabil. 1998;79(11):1473-76.

[PMID:9821913] http://dx.doi.org/10.1016/S0003-9993(98)90247-7

12. Nyland J, Bernasek T, Markee B, Dundore C. Comparison of the Easy Strutter Functional Orthosis System and axillary crutches during modified 3-point gait. J Rehabil Res 
Dev. 2004;41(2):195-206. [PMID:15558373]

http://dx.doi.org/10.1682/JRRD.2004.02.0195

13. Goh JC, Toh SL, Bose K. Biomechanical study on axillary crutches during single-leg swing-through gait. Prosthet Orthot Int. 1986;10(2):89-95. [PMID:3774516]

14. Stallard J, Dounis E, Major RE, Rose GK. One leg swing through gait using two crutches. An analysis of the ground reaction forces and gait phases. Acta Orthop Scand. 1980; 51(1):71-77. [PMID:7376848] http://dx.doi.org/10.3109/17453678008990771

15. Herman HH. Mobility assisting device. United States patent US 5,862,824. 1999.

16. Magee JA, Kenney DM. Use of strutter orthoses for an adolescent with myelodysplasia. Pediatr Phys Ther. 2008; 20(1):81-88. [PMID:18300937] http://dx.doi.org/10.1097/PEP.0b013e31815e4add

17. Sumiya T, Suzuki Y, Kasahara T, Ogata H. Sensing stability and dynamic response of the F-Scan in-shoe sensing system: a technical note. J Rehabil Res Dev. 1998;35(2): 192-200. [PMID:9651891]
18. Mueller MJ, Strube MJ. Generalizability of in-shoe peak pressure measures using the F-scan system. Clin Biomech (Bristol, Avon). 1996;11(3):159-64. [PMID:11415614] http://dx.doi.org/10.1016/0268-0033(95)00047-X

Submitted for publication January 20, 2012. Accepted in revised form July 19, 2012.

This article and any supplementary material should be cited as follows:

Borrelli J, Haslach HW Jr. Experimental characterization of axillary/underarm interface pressure in swing-through crutch walking. J Rehabil Res Dev. 2013;50(3):423-36. http://dx.doi.org/10.1682/JRRD.2012.01.0013

ResearcherID/ORCID: James Borrelli, PhD: C-7690-2011

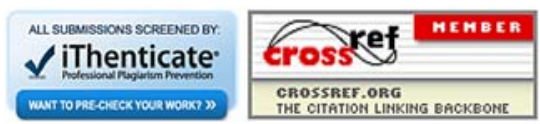


